\title{
Mechanical Proof of the Maxwell-Boltzmann Speed Distribution With Numerical Iterations
}

\author{
Hejie Lin ${ }^{1} \&$ Tsung-Wu Lin ${ }^{2}$ \\ ${ }^{1}$ Department of Mechanical Engineering, Oakland University, Rochester Hills, Michigan, USA \\ ${ }^{2}$ Department of Civil Engineering, National Taiwan University, Taipei, Taiwan \\ Correspondence: Hejie Lin, Department of Mechanical Engineering, Oakland University, Rochester Hills, Michigan, \\ USA. E-mail: hejielin@oakland.edu
}

Received: April 11, $2021 \quad$ Accepted: May 17, $2021 \quad$ Online Published: June 1, 2021

doi:10.5539/ijsp.v10n4p21 URL: https://doi.org/10.5539/ijsp.v10n4p21

\begin{abstract}
The Maxwell-Boltzmann speed distribution is the probability distribution that describes the speeds of the particles of ideal gases. The Maxwell-Boltzmann speed distribution is valid for both un-mixed particles (one type of particle) and mixed particles (two types of particles). For mixed particles, both types of particles follow the Maxwell-Boltzmann speed distribution. Also, the most probable speed is inversely proportional to the square root of the mass.

This paper proves the Maxwell-Boltzmann speed distribution and the speed ratio of mixed particles using computer-generated data based on Newton's law of motion. To achieve this, this paper derives the probability density function $\psi^{a b}\left(u_{a} ; v_{a}, v_{b}\right)$ of the speed $u_{a}$ of the particle with mass $M_{a}$ after the collision of two particles with mass $M_{a}$ in speed $v_{a}$ and mass $M_{b}$ in speed $v_{b}$. The function $\psi^{a b}\left(u_{a} ; v_{a}, v_{b}\right)$ is obtained through a unique procedure that considers (1) the randomness of the relative direction before a collision by an angle $\alpha$. (2) the randomness of the direction after the collision by another independent angle $\beta$.

The function $\psi^{a b}\left(u_{a} ; v_{a}, v_{b}\right)$ is used in the equation below for the numerical iterations to get new distributions $\mathrm{P}_{\text {new }}^{a}\left(u_{a}\right)$ from old distributions $\mathrm{P}_{\text {old }}^{a}\left(v_{a}\right)$, and repeat with $\mathrm{P}_{\text {old }}^{a}\left(v_{a}\right)=\mathrm{P}_{\text {new }}^{a}\left(v_{a}\right)$, where $\mathrm{n}_{a}$ is the fraction of particles with mass $\mathrm{M}_{a}$.

$$
\begin{aligned}
\mathrm{P}_{\text {new }}^{1}\left(u_{1}\right) & =\mathrm{n}_{1} \int_{0}^{\infty} \int_{0}^{\infty} \psi^{11}\left(u_{1} ; v_{1}, v_{1}^{\prime}\right) \mathrm{P}_{\text {old }}^{1}\left(v_{1}\right) \mathrm{P}_{\text {old }}^{1}\left(v_{1}^{\prime}\right) d v_{1} d v_{1}^{\prime} \\
& +\mathrm{n}_{2} \int_{0}^{\infty} \int_{0}^{\infty} \psi^{12}\left(u_{1} ; v_{1}, v_{2}\right) \mathrm{P}_{\text {old }}^{1}\left(v_{1}\right) \mathrm{P}_{\text {old }}^{2}\left(v_{2}\right) d v_{1} d v_{2} \\
\mathrm{P}_{\text {new }}^{2}\left(u_{2}\right) & =\mathrm{n}_{1} \int_{0}^{\infty} \int_{0}^{\infty} \psi^{21}\left(u_{2} ; v_{2}, v_{1}\right) \mathrm{P}_{\text {old }}^{2}\left(v_{2}\right) \mathrm{P}_{\text {old }}^{1}\left(v_{1}\right) d v_{2} d v_{1} \\
& +\mathrm{n}_{2} \int_{0}^{\infty} \int_{0}^{\infty} \psi^{22}\left(u_{2} ; v_{2}, v_{2}^{\prime}\right) \mathrm{P}_{\text {old }}^{2}\left(v_{2}\right) \mathrm{P}_{\text {old }}^{2}\left(v_{2}^{\prime}\right) d v_{2} d v_{2}^{\prime}
\end{aligned}
$$
\end{abstract}

The final distributions converge to the Maxwell-Boltzmann speed distributions. Moreover, the square of the root-mean-square speed from the final distribution is inversely proportional to the particle masses as predicted by Avogadro's law.

Keywords: Maxwell speed distribution, Maxwell-Boltzmann speed distribution, Maxwell-Boltzmann distribution, Avogadro's law, kinetic theory of gases, thermodynamics, statistical Mechanics, collisions of particles

\section{Overview}

James C. Maxwell (1860a,b) first provided the Maxwell speed distribution in 1860 on a statistical heuristic basis. Maxwell (1867) and Boltzmann (1872) carried out more investigations into the physical meaning of the distribution. Boltzmann (1877) derived the distribution again based on statistical thermodynamics. Nevertheless, none of their approaches were based on Newton's law of motion.

A way to prove the Maxwell-Boltzmann speed distribution is from a statistical view, beginning from the Boltzman distribution of the velocity $v_{x}$ in the x-direction as follows.

$$
P\left(v_{x}\right)=\frac{1}{\sigma \sqrt{2 \pi}} e^{\frac{-1}{2}\left(\frac{v_{x}}{\sigma}\right)^{2}}
$$

or 


$$
P\left(v_{x}\right)=\frac{h}{\sqrt{\pi}} e^{-h^{2} v_{x}^{2}}
$$

Where $\sigma=\frac{1}{\sqrt{2} h}, h=\sqrt{\frac{M}{2 k T}}, k$ is the Boltzmann constant, $T$ is the equilibrium temperature, and $M$ is the particle mass. Extending from the velocity $v_{x}$ to three independent velocities $\left(v_{x}, v_{y}, v_{z}\right)$ in three directions, and transferring it to spherical coordinates $(v, \theta, \varphi)$ using $v_{x}=v \sin \theta \cos \varphi, v_{y}=v \sin \theta \sin \varphi$, and $v_{z}=v \cos \theta$ gives

$$
\begin{gathered}
\int_{-\infty}^{\infty} \int_{-\infty}^{\infty} \int_{-\infty}^{\infty} P\left(v_{x}\right) P\left(v_{y}\right) P\left(v_{z}\right) d v_{x} d v_{y} d v_{z} \\
=\int_{0}^{\infty} \frac{4 h^{3}}{\sqrt{\pi}} v^{2} e^{-h^{2} v^{2}} d v=\int_{0}^{\infty} P(v) d v
\end{gathered}
$$

Where $P(v)$ is the Maxwell-Boltzmann speed distribution shown in Equation (4) (Brush, 1966, Landau et al., 1969, McQuarrie, 1976, Garrod, 1995, Maudlin, 2013).

$$
P(v)=\frac{4 h^{3}}{\sqrt{\pi}} v^{2} e^{-h^{2} v^{2}}
$$

In the Maxwell-Boltzmann speed distribution, the most probable speed, $v_{m p}$, is inversely proportional to the square root of the mass for fixed temperatures as follows

$$
v_{m p}=\frac{1}{h}=\sqrt{\frac{2 k T}{M}}
$$

Therefore, when two types of particles with mass $M_{1}$ and $M_{2}$ are mixed at the same temperature, the above equation gives the following mass-speed relationship

$$
\frac{v_{1, m p}}{v_{2, m p}}=\sqrt{\frac{M_{2}}{M_{1}}}
$$

An example of the theoretical Maxwell-Boltzmann speed distribution curves and their corresponding most probable speeds $v_{1, m p}$ and $v_{2, m p}$ of two types of particles with a mass ratio of nine are shown below.

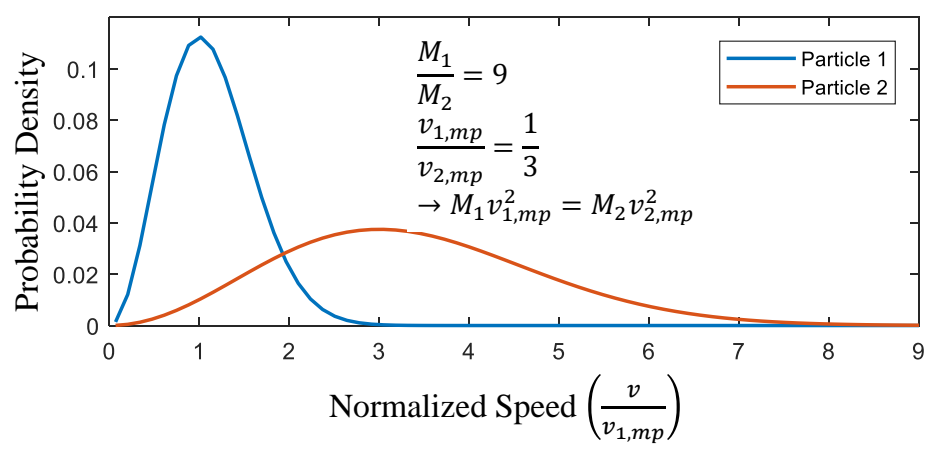

Figure 1. Maxwell-Boltzmann speed distributions of two types of particles

Boltzmann (1872) tried to provide mechanical proof of the Maxwell-Boltzmann speed distribution in 1872 by formulating the following equation.

$$
d n=f(x, t) d x \cdot f\left(x^{\prime}, t\right) d x^{\prime} \cdot \psi\left(\xi ; x, x^{\prime}\right) d \xi
$$

where $f(x, t) d x$ is the number of particles with speeds between $x$ and $x+d x$, and similarly for $f\left(x^{\prime}, t\right) d x^{\prime}, d n$ is the number of particles with speeds between $\xi$ and $\xi+d \xi$. In addition, the symbol $\psi\left(\xi ; x, x^{\prime}\right)$ represents the probability density function (PDF) of the resulting speed after a collision between two particles. The definition is excellent and meaningful, but the method used to calculate this PDF $\psi$ has yet to be created. Following Boltzmann's work in 1872, we derived the PDF $\psi$ based on Newton's law of motion in this paper. The PDF $\psi$ for equal mass particles had been provided by Lin et al. (2019). 
To consider the collisions of unequal mass particles, we need to have four PDFs: $\psi^{11}\left(u_{1} ; v_{1}, v_{1}^{\prime}\right), \psi^{12}\left(u_{1} ; v_{1}, v_{2}\right)$, $\psi^{21}\left(u_{2} ; v_{2}, v_{1}\right)$, and $\psi^{22}\left(u_{2} ; v_{2}, v_{2}^{\prime}\right)$. Where $\psi^{a b}\left(u_{a} ; v_{a}, v_{b}\right)$ is the PDF of post-collision speed $u_{a}$ of a particle with a mass $M_{a}$ after the collision of two particles with mass $M_{a}$ in a pre-collision speed $v_{a}$ and mass $M_{b}$ in a pre-collision speed $v_{b}$. For $a=b$, the PDF is identical to the collision of two equal mass particles. For $a \neq b$, the PDF will be given in an integral form that will later be evaluated numerically for collision angles from 0 to $\pi$.

After the PDF $\psi^{a b}\left(u_{a} ; v_{a}, v_{b}\right)$ is derived, numerical iterations are used to get new distributions $\mathrm{P}_{\text {new }}^{a}\left(u_{a}\right)$ from old distributions $\mathrm{P}_{\text {old }}^{a}\left(v_{a}\right)$, and set $\mathrm{P}_{\text {old }}^{a}\left(v_{a}\right)=\mathrm{P}_{\text {new }}^{a}\left(v_{a}\right)$ for the next iteration, where $\mathrm{n}_{a}$ is the fraction of particles with mass $M_{a}$ using the following equations.

$$
\begin{aligned}
\mathrm{P}_{\text {new }}^{1}\left(u_{1}\right) & =\mathrm{n}_{1} \int_{0}^{\infty} \int_{0}^{\infty} \psi^{11}\left(u_{1} ; v_{1}, v_{1}^{\prime}\right) \mathrm{P}_{\text {old }}^{1}\left(v_{1}\right) \mathrm{P}_{\text {old }}^{1}\left(v_{1}^{\prime}\right) d v_{1} d v_{1}^{\prime} \\
& +\mathrm{n}_{2} \int_{0}^{\infty} \int_{0}^{\infty} \psi^{12}\left(u_{1} ; v_{1}, v_{2}\right) \mathrm{P}_{\text {old }}^{1}\left(v_{1}\right) \mathrm{P}_{\text {old }}^{2}\left(v_{2}\right) d v_{1} d v_{2} \\
\mathrm{P}_{\text {new }}^{2}\left(u_{2}\right) & =\mathrm{n}_{1} \int_{0}^{\infty} \int_{0}^{\infty} \psi^{21}\left(u_{2} ; v_{2}, v_{1}\right) \mathrm{P}_{\text {old }}^{2}\left(v_{2}\right) \mathrm{P}_{\text {old }}^{1}\left(v_{1}\right) d v_{2} d v_{1} \\
& +\mathrm{n}_{2} \int_{0}^{\infty} \int_{0}^{\infty} \psi^{22}\left(u_{2} ; v_{2}, v_{2}^{\prime}\right) \mathrm{P}_{\text {old }}^{2}\left(v_{2}\right) \mathrm{P}_{\text {old }}^{2}\left(v_{2}^{\prime}\right) d v_{2} d v_{2}^{\prime}
\end{aligned}
$$

Note that $\mathrm{n}_{1}+\mathrm{n}_{2}=1$. After about thirteen iterations, the final distributions converge to the Maxwell-Boltzmann speed distributions. Moreover, the square of the RMS speed is inversely proportional to the particle masses, i.e. $M_{1} v_{1, r m s}^{2}=M_{2} v_{2, r m s}^{2}$. For the same pressure, from the kinetic theory of ideal gases (Herapath, 1821, Waterston, 1843:1892, Lane, 2003), $p_{1}=\frac{1}{3} \rho_{1} v_{1, \text { rms }}^{2}=\frac{1}{3} \rho_{2} v_{2, r m s}^{2}=p_{2}$. Where the densities $\rho_{1}=M_{1} / V_{1}$ and $\rho_{2}=M_{2} / V_{2}$. Therefore, it can be concluded that the volumes occupied for each particle, $V_{1}$ or $V_{2}$, are the same, $V_{1}=V_{2}$, as Avogadro's law predicted (Avogadro, 1811). In this sense, it also gives a mechanical proof of Avogadro's law.

\section{Velocity Diagram for Collision of Two Particles}

A velocity diagram for a collision of two particles is used to derive the probability density function. Two concentric circles in the 2D plane can be constructed as a velocity diagram for a collision of two particles in 3D space. The concentric circles velocity diagram provides a geometric relationship between the pre-collision and post-collision speeds of a collision. The concentric circles velocity diagram was used by Maxwell in his study of the Maxwell-Boltzmann speed distribution and is explained and proved in this section.

Concentric circles velocity diagrams are based on two reference frames: a fixed reference frame (O) and a center-of-mass $(\mathrm{CM})$ reference frame $(\mathrm{C})$. Speeds can be transferred between the fixed frame $(\mathrm{O})$ and the CM frame (C).

\subsection{The Fixed Reference Frame}

In the fixed reference frame, before a collision, two particles with mass $M_{1}$ and $M_{2}$ are moving at pre-collision velocities $\vec{v}_{1}$ (or $\overrightarrow{O A}$ ) and $\vec{v}_{2}$ (or $\overrightarrow{O B}$ ). After the collision, the post-collision velocities of the two particles change to $\vec{u}_{1}$ (or $\overrightarrow{O P}$ ) and $\vec{u}_{2}$ (or $\overrightarrow{O Q}$ ), as shown in Figure 2. It is important to note that the variables in the PDF $\psi^{12}\left(u_{1} ; v_{1}, v_{2}\right)$ are speeds, which are the magnitudes of the velocities. Note that the vector $\vec{v}$ without an arrow-hat $v$ indicates the length of the vector.

For a perfectly elastic collision, based on Newton's law of motion, both total momentum and total energy remain unchanged before and after a collision as following

$$
\begin{aligned}
\text { Momentum } & =M_{1} \vec{v}_{1}+M_{2} \vec{v}_{2}=M_{1} \vec{u}_{1}+M_{2} \vec{u}_{2} \\
\text { Total Energy } & =\left(M_{1} v_{1}^{2}+M_{2} v_{2}^{2}\right) / 2=\left(M_{1} u_{1}^{2}+M_{2} u_{2}^{2}\right) / 2
\end{aligned}
$$




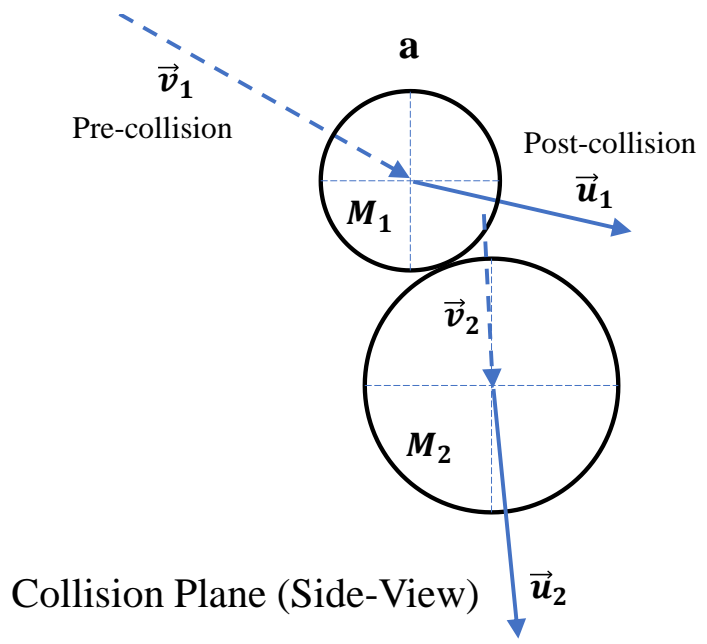

b

\section{Fixed Frame $(\mathrm{O})$}

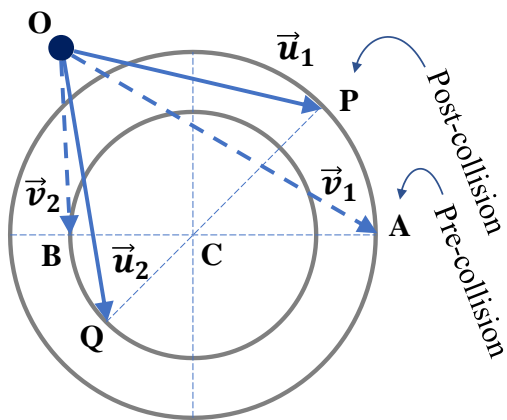

Concentric Circles Diagram

Figure 2. Velocities of two particles before $\left(\vec{v}_{1}, \vec{v}_{2}\right)$ and after $\left(\vec{u}_{1}, \vec{u}_{2}\right)$ a collision

\subsection{The Concentric Circles in Center-of-Mass Reference Frame}

The center-of-mass (CM) reference frame uses the center of mass of two particles as its origin point (C). The velocity of the center-of-mass of two particles is related to the two masses $M_{1}$ and $M_{2}$ and their corresponding velocities $\vec{v}_{1}$ and $\vec{v}_{2}$ as

$$
\vec{v}_{c} \equiv \frac{M_{1} \vec{v}_{1}+M_{2} \vec{v}_{2}}{M_{1}+M_{2}} \equiv m_{1} \vec{v}_{1}+m_{2} \vec{v}_{2}
$$

where $m_{1}$ and $m_{2}$ are mass ratio and are defined as: $m_{1} \equiv \frac{M_{1}}{M_{1}+M_{2}} ; m_{2} \equiv \frac{M_{2}}{M_{1}+M_{2}}$.

Before the collision, the pre-collision velocities of particles 1 and 2 in the CM frame are

$$
\begin{aligned}
& \vec{v}_{1 c} \equiv \vec{v}_{1}-\vec{v}_{c} \\
& \vec{v}_{2 c} \equiv \vec{v}_{2}-\vec{v}_{c}
\end{aligned}
$$

Based on the conservation of momentum, the total momentum in the CM frame is zero. Since the CM frame is an inertial reference frame moving with the constant velocity $\vec{v}_{c}$ and the particle velocities are relative to the CM frame,

$$
m_{1} \vec{v}_{1 c}+m_{2} \vec{v}_{2 c}=0
$$

The above equation guarantees two things: (1) Point $\mathrm{C}$ will be located in a straight line between point $\mathrm{A}$ and point $\mathrm{B}$, and (2) the distance ratio from the center-of-mass point $C$ to point $A$ and point $B$ is

$$
\frac{v_{1 c}}{v_{2 c}}=\frac{m_{2}}{m_{1}}
$$

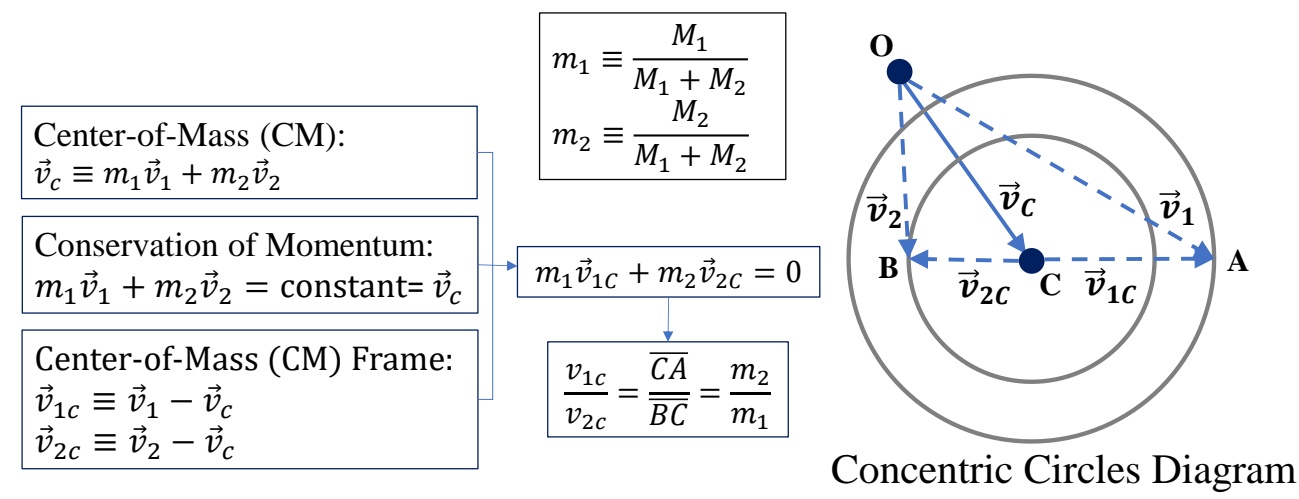

Figure 3. Pre-collision velocities of two particles relative to center-of-mass 
In the CM frame, two concentric circles can be constructed:

Circle $\mathrm{C}-\mathrm{A}$ : a circle center at point $\mathrm{C}$ with a radius of $|\overrightarrow{C A}|$

Circle C-B: a circle center at point $\mathrm{C}$ with a radius of $|\overrightarrow{C B}|$

Using these two concentric circles in the CM frame, constraints of the post-collision velocities can be formulated and visualized as shown in Figure 4.

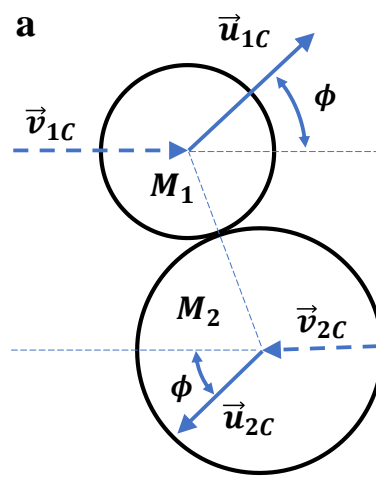

$$
\frac{u_{1 c}}{u_{2 c}}=\frac{v_{1 c}}{v_{2 c}}=\frac{\overline{C A}}{\overline{B C}}=\frac{m_{2}}{m_{1}}
$$

b

\section{Center-of-Mass Frame (C)}

\section{Concentric Circles Diagram}

Figure 4. Post-collision velocities $\left(\vec{u}_{1 c}, \vec{u}_{2 c}\right)$ of two particles relative to center-of-mass

After the collision, the post-collision velocities of particle 1 and particle 2 change to $\vec{u}_{1 c}$ (or $\overrightarrow{C P}$ ) and $\vec{u}_{2 c}($ or $\overrightarrow{C Q}$ ) according to the following three rules:

1. Point $\mathrm{P}$ will be on Circle C-A. $\therefore u_{1 c}=v_{1 c}$

2. Point Q will be on Circle C-B. $\therefore u_{2 c}=v_{2 c}$ and $\frac{u_{1 c}}{u_{2 c}}=\frac{|\overrightarrow{C P}|}{|\overrightarrow{C Q}|}=\frac{|\overrightarrow{C A}|}{|\overrightarrow{C B}|}=\frac{m_{2}}{m_{1}}$

3. Velocity $\overrightarrow{C P}$ and $\overrightarrow{C Q}$ have opposite directions. $\therefore m_{1} \vec{u}_{1 c}+m_{2} \vec{u}_{2 c}=0$

The post-collision velocities based on the above three rules satisfy the (1) conservation of momentum and (2) conservation of energy, as shown in Equations (14) and (15) and Figure 5.

Conservation of Momentum:

$$
\mathrm{m}_{1}\left(\vec{v}_{c}+\vec{u}_{1 c}\right)+\mathrm{m}_{2}\left(\vec{v}_{c}+\vec{u}_{2 c}\right)=\vec{v}_{c}
$$

Conservation of Energy:

$$
\begin{gathered}
\mathrm{m}_{1}\left(\vec{v}_{c}+\vec{u}_{1 c}\right) \cdot\left(\vec{v}_{c}+\vec{u}_{1 c}\right)+\mathrm{m}_{2}\left(\vec{v}_{c}+\vec{u}_{2 c}\right) \cdot\left(\vec{v}_{c}+\vec{u}_{2 c}\right) \\
=v_{c}^{2}+\mathrm{m}_{1} u_{1 c}^{2}+\mathrm{m}_{2} u_{2 c}^{2}=v_{c}^{2}+\mathrm{m}_{1} v_{1 c}^{2}+\mathrm{m}_{2} v_{2 c}^{2}
\end{gathered}
$$

\section{Concentric Circles in the Center-of-Mass Frame (C)}
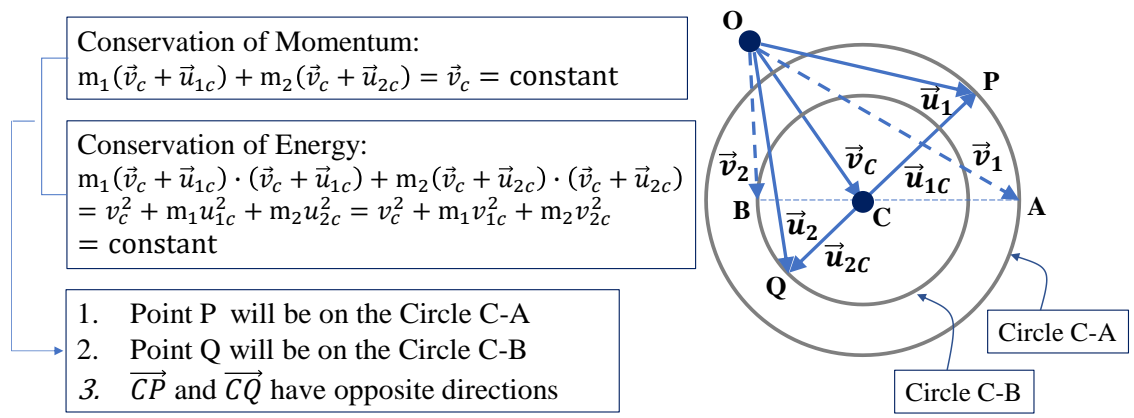

Figure 5. Post-collision velocities $\left(\vec{u}_{1 c}, \vec{u}_{2 c}\right)$ from conservations of momentum and energy 


\section{Derivation of the Probability Density Function $\Psi^{12}$}

After any collision between two particles, the resulting speeds depend on two factors: (1) the random directions of the two pre-collision velocities $\vec{v}_{1}$ and $\vec{v}_{2}$ and (2) the random direction of the post-collision velocity $\vec{u}_{1 C}$, which is related to the contact point of the two particles. These two random factors are used in the following equation for the derivation of the PDF $\psi^{12}$.

$$
\Psi^{12}\left(u_{1} ; v_{1}, v_{2}\right)=\int_{0}^{\pi} \mathrm{P}_{u_{1} \mid \alpha}\left(u_{1}\right) \mathrm{P}_{\alpha}(\alpha) d \alpha=\int_{0}^{\pi} \mathrm{P}_{\beta \mid \alpha}(\beta)\left|\frac{d \beta}{d u_{1}}\right| \mathrm{P}_{\alpha}(\alpha) d \alpha
$$

Where $\alpha$ is the angle between $\vec{v}_{1}$ and $\vec{v}_{2}$, and $\beta$ is the angle between $\vec{v}_{C}$ and $\vec{u}_{1 C}$.

The right-hand side of the above equation will be derived in the following sections. The final formula of the probability density function is shown in Equation (24).

\subsection{The Randomness of the Directions in 3D Before a Collision}

For two given pre-collision speeds $v_{1}$ and $v_{2}$, the post-collision speeds depend on the directions of the two pre-collision velocities $\vec{v}_{1}$ and $\vec{v}_{2}$. The two pre-collision velocities both have random directions. The directions of these two pre-collision velocities determine the radius of circle $\mathrm{C}$-A and circle $\mathrm{C}-\mathrm{B}$. The randomness of the two velocities can be reduced to one random angle $\alpha$ which considers only the relative direction between $\vec{v}_{1}$ and $\vec{v}_{2}$ and is defined as the angle between the two pre-collision velocities $\vec{v}_{1}$ and $\vec{v}_{2}$ (in the fixed frame) as shown.

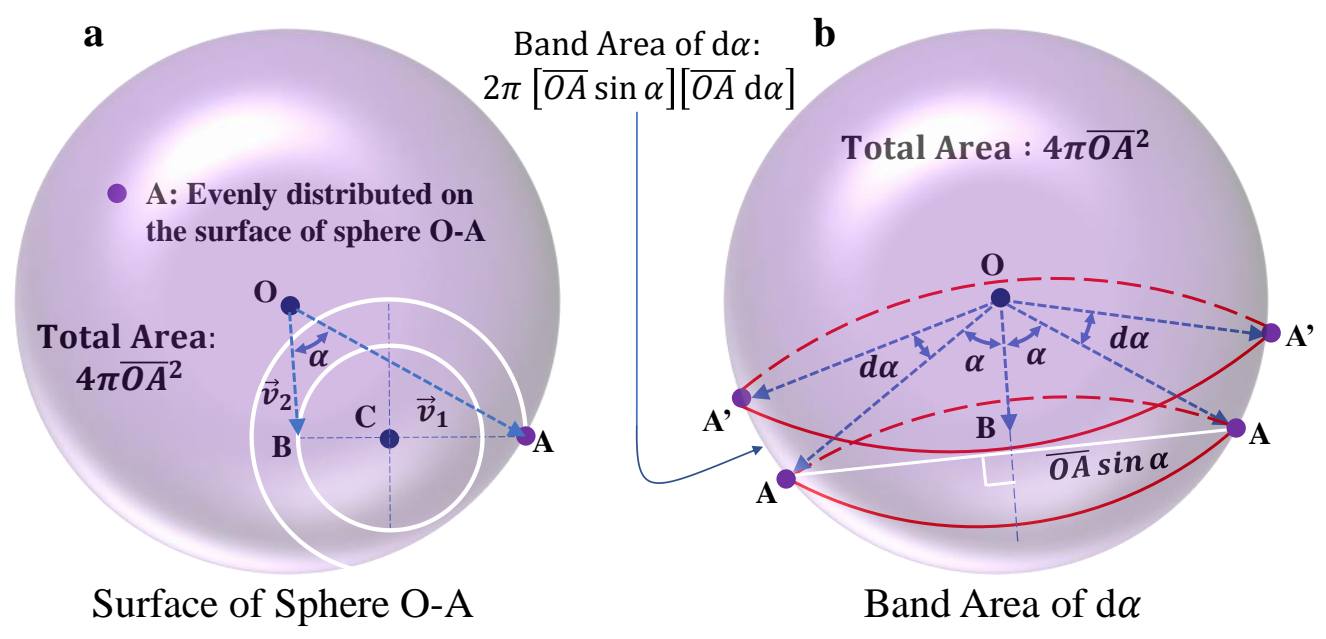

Figure 6. Band area of $d \alpha$ for the probability of the angle $\alpha$

For fixed magnitudes of $v_{1}$ and $v_{2}$, if $\vec{v}_{2}=\overline{O B}$ is also fixed in the direction, but the direction of $\vec{v}_{1}=\overline{O A}$ is changed, then point A will be located on a spherical surface O-A, as shown in Figure 6 . And the probability of point A on the surface is uniformly distributed since $\vec{v}_{1}$ has equal opportunity in any direction. Therefore the velocity vector of mass 1 before the collision has a random direction in 3D space. The sphere surface $\mathrm{O}-\mathrm{A}$ is centered at point $\mathrm{O}$ and its radius is $v_{1}$. The band area between $\alpha$ and $\alpha+\mathrm{d} \alpha$ on the sphere surface O-A is $2 \pi\left(v_{1} \sin \alpha\right)\left(v_{1} \mathrm{~d} \alpha\right)$, the total area of the sphere surface is $4 \pi v_{1}^{2}$, and the ratio is $\frac{1}{2} \sin \alpha d \alpha$. Therefore the probability density of point A located on the sphere surface $\mathrm{O}-\mathrm{A}$ at angle $\alpha$ is

$$
\mathrm{P}_{\alpha}(\alpha)=\frac{1}{2} \sin \alpha
$$




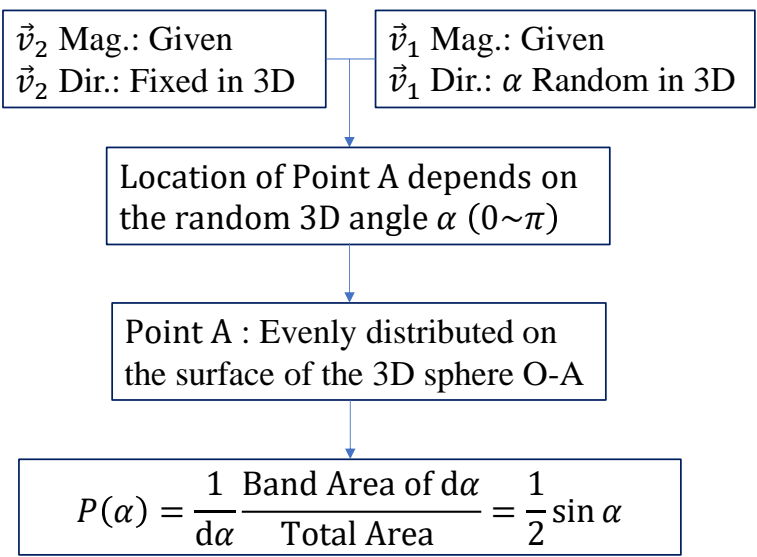

3.2 The Randomness of the Directions in 3D after a Collision

Besides the pre-collision directions defined by $\alpha$ (representing the relative moving direction before the collision), the location of the contact point also determines the post-collision velocity. The random location of the contact point is related to the location of Mass 1 center before the collision.

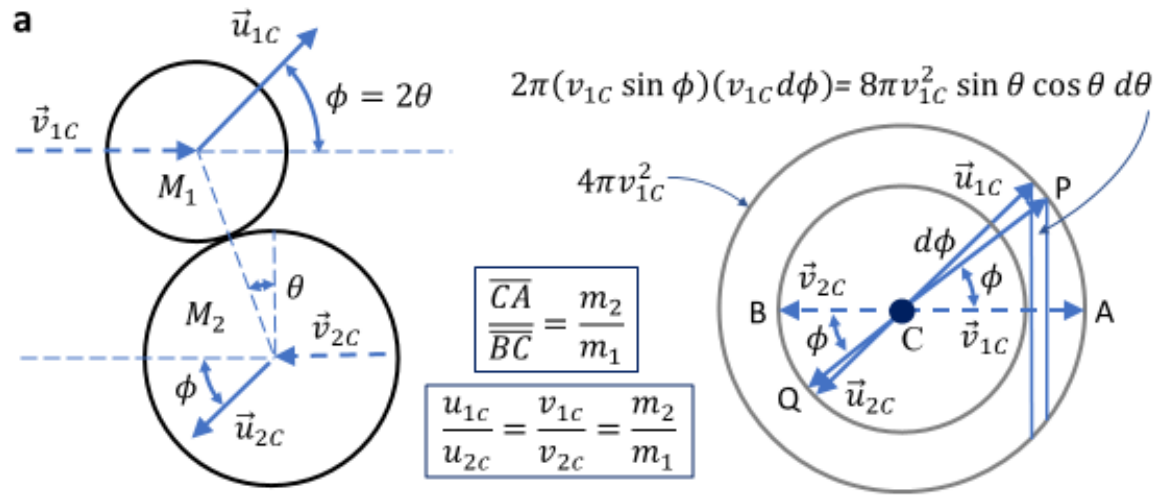

\section{Collision Plane (Side-View)}

Concentric Sphere Surfaces

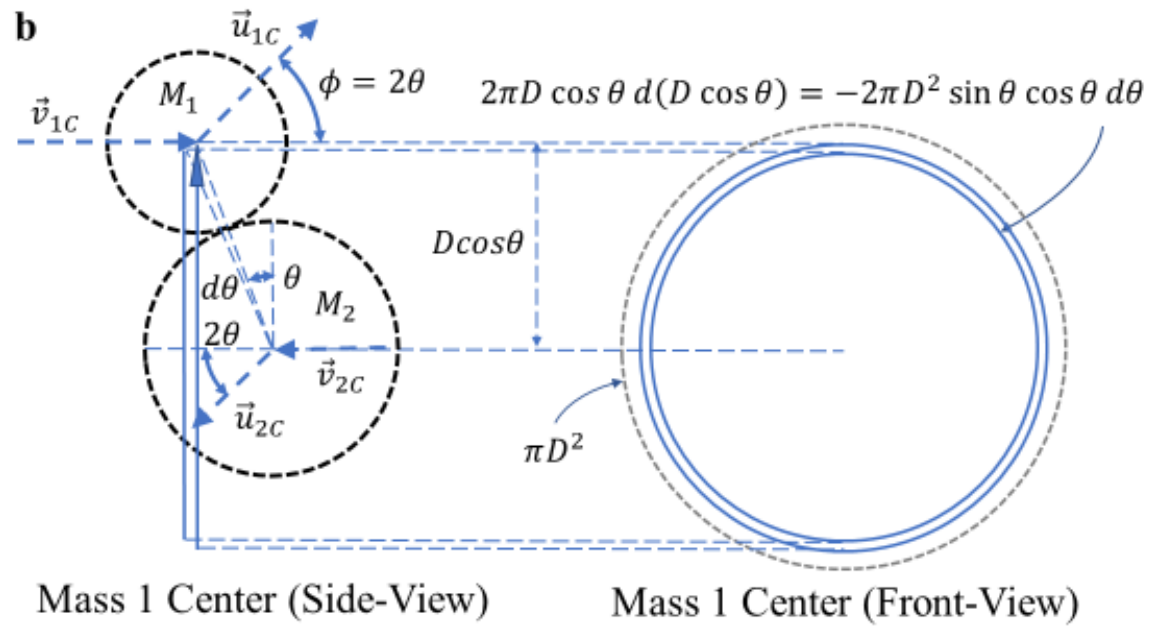

Figure 7. (a) Band area of $d \phi$ for the post-collision velocity $\vec{u}_{1 C}$,

(b) Ring area of $d \theta$ for the location of mass 1 center

It can be observed that Point $\mathrm{P}$ is always located on the sphere surface C-P and that the probability is uniform on the 
surface. The reasons are based on the following factors:

(1) The band-area in Figure 7(a) is $8 \pi v_{1 C}^{2} \sin \theta \cos \theta d \theta$.

(2) The ring-area in Figure 7(b) is $2 \pi D^{2} \sin \theta \cos \theta d \theta$.

(3) The ratio of the two areas is $4 v_{1 C}^{2} / D^{2}$, it is not dependent on $\theta$.

(4) When the Center of Mass 1 hits inside the ring of the disk, Point $\mathrm{P}$ will be located inside the band on the sphere surface.

(5) It has an equal opportunity to hit any point inside the disk.

Similar to the pre-collision directions defined by $\alpha$ (representing the relative moving direction before the collision), the location of Point $\mathrm{P}$ will be defined by $\beta$, as the angle between $\vec{v}_{C}$ and $\vec{u}_{1 C}$, as shown in Figure 8 , such that the same angle $\beta$ will result in the same magnitude of the different velocity $\vec{u}_{1}$.

$\mathbf{a}$

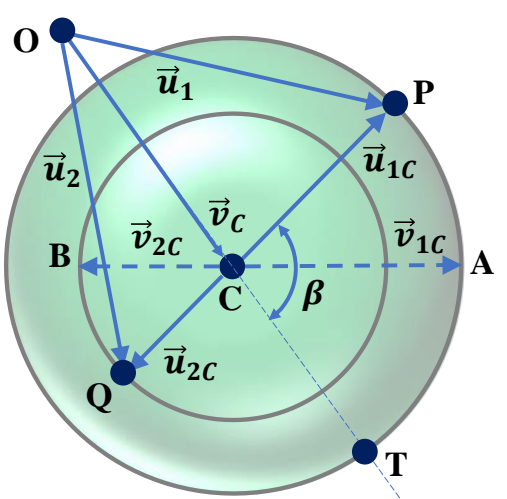

Surface of Sphere C-A b Band Area of $\mathrm{d} \beta: 2 \pi[\overline{C P} \sin \beta][\overline{C P} \mathrm{~d} \beta]$ )

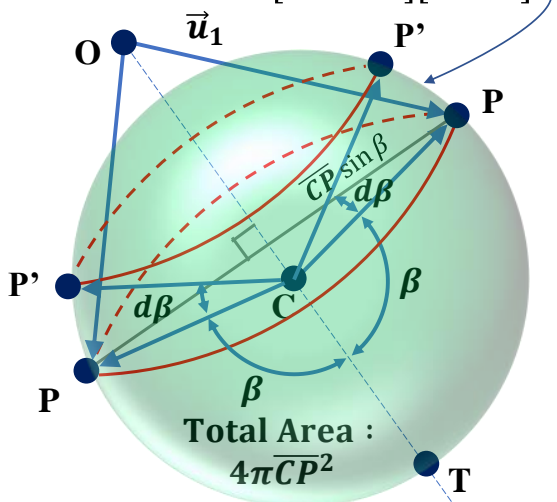

Band Area of $\mathrm{d} \beta$

Figure 8. Band area of $\mathrm{d} \beta$ for the probability of the angle $\beta$

The band area between $\beta$ and $\beta+\mathrm{d} \beta$ on the sphere surface C-P is $2 \pi\left(v_{1 C} \sin \beta\right)\left(v_{1 C} \mathrm{~d} \beta\right)$, the total area of the sphere surface is $4 \pi v_{1 C}^{2}$, and the ratio is $\frac{1}{2} \sin \beta d \beta$. Therefore the probability density of Point P located on the sphere surface $\mathrm{C}-\mathrm{P}$ at angle $\beta$ is

$$
\mathrm{P}_{\beta \mid \alpha}(\beta)=\frac{1}{2} \sin \beta
$$

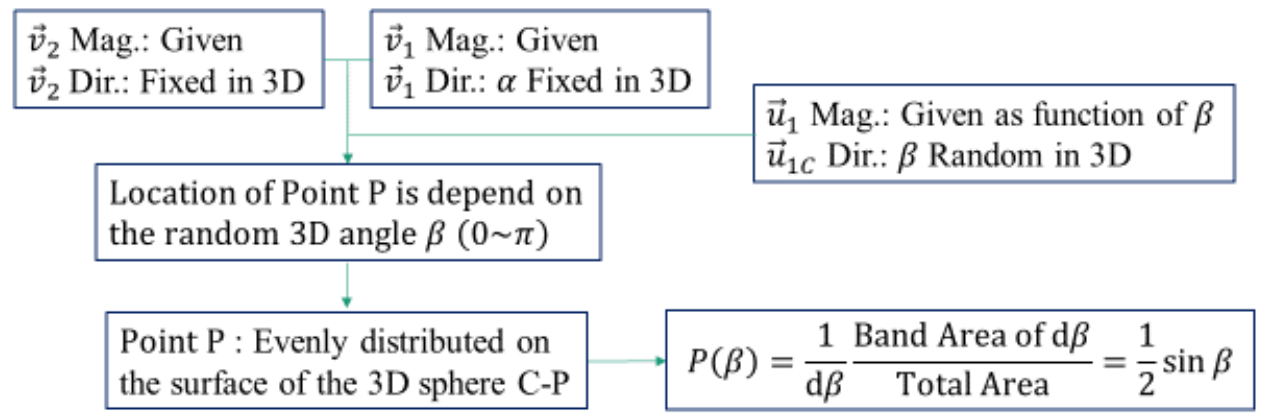

\subsection{Considering all the Possible Directions in 3D Before and After Collisions}

The $r_{1}$ and $r_{2}$ as shown in Figure 9 can be computed from $\alpha$ as 


$$
\begin{aligned}
r_{1}\left(\alpha ; v_{1}, v_{2}\right)=\overline{O C} & =\sqrt{\left(m_{1} v_{1} \cos \alpha+m_{2} v_{2}\right)^{2}+\left(m_{1} v_{1} \sin \alpha\right)^{2}} \\
& =m_{1} \sqrt{v_{1}^{2}+\left(\frac{m_{2}}{m_{1}} v_{2}\right)^{2}+2 v_{1}\left(\frac{m_{2}}{m_{1}} v_{2}\right) \cos \alpha} \\
r_{2}\left(\alpha ; v_{1}, v_{2}\right)=\overline{B C} & =\sqrt{\left(m_{1} v_{1} \cos \alpha-m_{1} v_{2}\right)^{2}+\left(m_{1} v_{1} \sin \alpha\right)^{2}} \\
& =m_{1} \sqrt{v_{1}^{2}+v_{2}^{2}-2 v_{1} v_{2} \cos \alpha}
\end{aligned}
$$

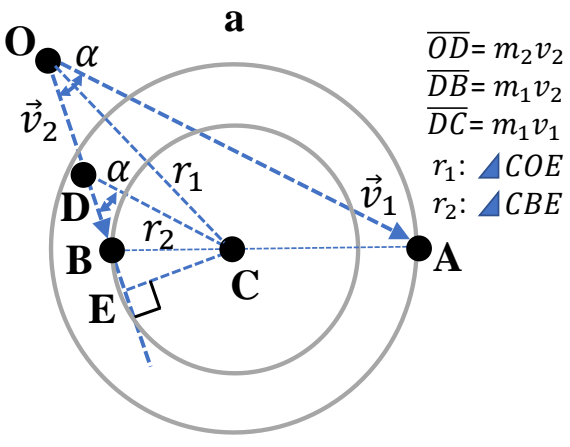

Relation Between $\left(r_{1}, r_{2}\right)$ and $\left(\alpha, v_{1}, v_{2}\right)$

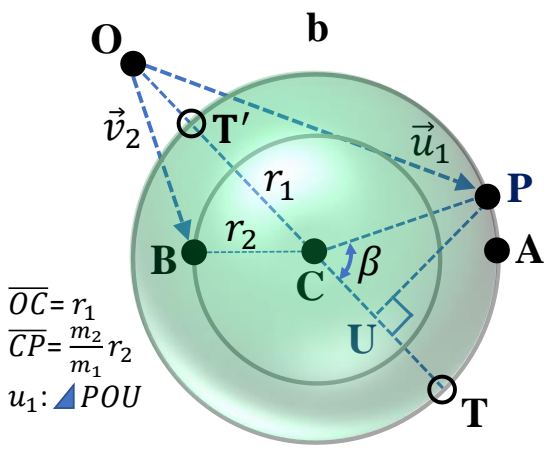

Relation Between $u_{1}$ and $\left(\beta, r_{1}, r_{2}\right)$

Figure 9. Relations for $r_{1}, r_{2}$ and $u_{1}$

So the relation between $u_{1}$ and $\beta$ for fixed $r_{1}$ and $r_{2}$ is given by

$$
\begin{aligned}
u_{1}\left(\beta ; r_{1}, r_{2}\right)=\overline{O P} & =\sqrt{\left(\frac{m_{2}}{m_{1}} r_{2} \cos \beta+r_{1}\right)^{2}+\left(\frac{m_{2}}{m_{1}} r_{2} \sin \beta\right)^{2}} \\
& =\sqrt{r_{1}^{2}+\left(\frac{m_{2}}{m_{1}} r_{2}\right)^{2}+2 r_{1}\left(\frac{m_{2}}{m_{1}} r_{2}\right) \cos \beta}
\end{aligned}
$$

Hence

$$
\frac{d u_{1}}{d \beta}=\frac{-r_{1}\left(\frac{m_{2}}{m_{1}} r_{2}\right) \sin \beta}{\sqrt{r_{1}^{2}+\left(\frac{m_{2}}{m_{1}} r_{2}\right)^{2}+2 r_{1}\left(\frac{m_{2}}{m_{1}} r_{2}\right) \cos \beta}}=\frac{-r_{1}\left(\frac{m_{2}}{m} r_{2}\right) \sin \beta}{u_{1}}
$$

Use the relation above to change $\mathrm{P}_{\beta \mid \alpha}(\beta)=\frac{1}{2} \sin \beta$ into

$$
\mathrm{P}_{u_{1} \mid \alpha}\left(u_{1}\right)=\mathrm{P}_{\beta \mid \alpha}(\beta)\left|\frac{d \beta}{d u_{1}}\right|=\frac{u_{1}}{2 r_{1}\left(\frac{m_{2}}{m_{1}} r_{2}\right)}
$$

The PDF $\psi^{12}$ of post-collision speed $u_{1}$ for two given pre-collision speeds $v_{1}$ and $v_{2}$ can be obtained by summating all the densities for all the possible directions, i.e., $\alpha$ between 0 and $\pi$, yields

$$
\begin{gathered}
\Psi^{12}\left(u_{1} ; v_{1}, v_{2}\right)=\int_{0}^{\pi} \mathrm{P}_{u_{1} \mid \alpha}\left(u_{1}\right) \mathrm{P}_{\alpha}(\alpha) d \alpha \\
=\int_{0}^{\pi} \mathrm{P}_{\beta \mid \alpha}(\beta)\left|\frac{d \beta}{d u_{1}}\right| \mathrm{P}_{\alpha}(\alpha) d \alpha=\int_{0}^{\pi} \frac{u_{1}}{4 r_{1}\left(\frac{m_{2}}{m_{1}} r_{2}\right)} \sin \alpha d \alpha \\
=\int_{0}^{\pi} \frac{u_{1} \sin \alpha d \alpha}{4 m_{1} m_{2} \sqrt{v_{1}^{2}+\left(\frac{m_{2}}{m_{1}} v_{2}\right)^{2}+2 v_{1}\left(\frac{m_{2}}{m_{1}} v_{2}\right) \cos \alpha \sqrt{v_{1}^{2}+v_{2}^{2}-2 v_{1} v_{2} \cos \alpha}}} \\
=\frac{u_{1}}{4 m_{1} m_{2}} \int_{0}^{\pi} \frac{\sin \alpha d \alpha}{\sqrt{C+2 B \cos \alpha-A \cos ^{2} \alpha}}
\end{gathered}
$$


Where

$$
\begin{gathered}
A=4 v_{1}^{2} \hat{v}_{2}^{2}=r^{4} \sin ^{2}(2 \theta) \\
B=v_{1}\left(\frac{m_{2}}{m_{1}} v_{2}\right)\left(v_{1}^{2}+v_{2}^{2}\right)-v_{1} v_{2}\left[v_{1}^{2}+\left(\frac{m_{2}}{m_{1}} v_{2}\right)^{2}\right] \\
=2 v_{1} \hat{v}_{2}\left(v_{1}^{2}-\hat{v}_{2}^{2}\right)\left(\frac{m_{2}-m_{1}}{\sqrt{4 m_{1} m_{2}}}\right)=r^{4} \sin (2 \theta) \cos (2 \theta) \tan \varphi \\
C=\left[v_{1}^{2}+\left(\frac{m_{2}}{m_{1}} v_{2}\right)^{2}\right]\left(v_{1}^{2}+v_{2}^{2}\right) \\
\hat{v}_{2}=\sqrt{\frac{m_{2}}{m_{1}}} v_{2}, \quad r=\sqrt{v_{1}^{2}+\hat{v}_{2}^{2}}, \quad \theta=\tan ^{-1}\left(\frac{\hat{v}_{2}}{v_{1}}\right) \\
\varphi=\tan ^{-1}\left(\frac{m_{2}-m_{1}}{\sqrt{4 m_{1} m_{2}}}\right), \quad m_{1}=\frac{M_{1}}{M_{1}+M_{2}}, \quad m_{2}=\frac{M_{2}}{M_{1}+M_{2}}
\end{gathered}
$$

\section{Computational Proof by Numerical Iterations}

Both the Maxwell-Boltzmann speed distribution and the speed ratio of two types of particles can be validated using computer-generated data based on the PDF derived in the previous section. The detailed methodology and results are shown below.

\subsection{Setup of the Numerical Iterations}

Assume that particle type 1 has $\mathrm{n}_{1} N$ particles with mass $M_{1}$ and particle type 2 has $n_{2} N$ particles with mass $M_{2}$. For this example: it is given that $\left(\mathrm{n}_{1}, \mathrm{n}_{2}\right) N=(0.7,0.3) N,\left(M_{1}, M_{2}\right)=(0.9,0.1) M$. Note that the total number of particles and the particles' exact mass are irrelevant to the results. Also, the size of the monotonic particle is irrelevant to the results. The colliding speeds are discretized into 81 equally spaced speeds beginning from $v_{a}^{1}=0.5$ in increments of 1.0 and ending at $v_{a}^{81}=80.5$. The probabilities are assumed to be zero for speeds over 80.5.

\subsection{Formulas Used for the Iterations}

The equilibrium speed distribution can be obtained by numerical iterations. For discrete speeds, $v_{a}^{i}, i=1,2,3, \ldots, 81$, the Particle 1 probability $\mathrm{P}_{\text {new }}^{1}$ of the post-collision speed $u_{1}^{i}$ and the Particle 2 probability $\mathrm{P}_{\text {new }}^{2}$ of the post-collision speed $u_{2}^{i}$ after the collisions are:

$$
\begin{aligned}
\mathrm{P}_{\text {new }}^{1}\left(u_{1}^{i}\right) & =\mathrm{n}_{1} \sum_{j=1}^{81} \sum_{k=1}^{81} \psi^{11}\left(u_{1}^{i} ; v_{1}^{j}, v_{1}^{k}\right) \mathrm{P}_{\text {old }}^{1}\left(v_{1}^{j}\right) \mathrm{P}_{\text {old }}^{1}\left(v_{1}^{k}\right) \\
& +\mathrm{n}_{2} \sum_{j=1}^{81} \sum_{k=1}^{81} \psi^{12}\left(u_{1}^{i} ; v_{1}^{j}, v_{2}^{k}\right) \mathrm{P}_{\text {old }}^{1}\left(v_{1}^{j}\right) \mathrm{P}_{\text {old }}^{2}\left(v_{2}^{k}\right) \\
\mathrm{P}_{\text {new }}^{2}\left(u_{2}^{i}\right)= & \mathrm{n}_{1} \sum_{j=1}^{81} \sum_{k=1}^{81} \psi^{21}\left(u_{2}^{i} ; v_{2}^{j}, v_{1}^{k}\right) \mathrm{P}_{\text {old }}^{2}\left(v_{2}^{j}\right) \mathrm{P}_{\text {old }}^{1}\left(v_{1}^{k}\right) \\
& +\mathrm{n}_{2} \sum_{j=1}^{81} \sum_{k=1}^{81} \psi^{22}\left(u_{2}^{i} ; v_{2}^{j}, v_{2}^{k}\right) \mathrm{P}_{\text {old }}^{2}\left(v_{2}^{j}\right) \mathrm{P}_{\text {old }}^{2}\left(v_{2}^{k}\right)
\end{aligned}
$$

\subsection{Initial Distribution and Methodology of Iterations}

If we assume that the initial Root-Mean-Square speed is 10.5 and that the initial pre-collision speeds of all particles are 10.5 , that is $\mathrm{P}_{\text {old }}^{1}\left(v_{1}^{11}\right)=\mathrm{P}_{\text {old }}^{2}\left(v_{2}^{11}\right)=1$ and all others $\mathrm{P}_{\text {old }}^{1}\left(v_{1}^{i}\right)=\mathrm{P}_{\text {old }}^{2}\left(v_{2}^{i}\right)=0$, for $i \neq 11$. Use the equations above to get $\mathrm{P}_{\text {new }}^{1}\left(u_{1}^{i}\right)$ and $\mathrm{P}_{\text {new }}^{2}\left(u_{2}^{i}\right)$, and set $\mathrm{P}_{\text {old }}^{1}\left(v_{1}^{i}\right)=\mathrm{P}_{\text {new }}^{1}\left(v_{1}^{i}\right)$ and $\mathrm{P}_{\text {old }}^{2}\left(v_{2}^{i}\right)=\mathrm{P}_{\text {new }}^{2}\left(v_{2}^{i}\right)$ for the next iteration.

\subsection{Results of Iterations}

After thirteen iterations, both distribution curves converge to the Maxwell-Boltzmann speed distribution, as shown in Figure 10. Moreover, the square of RMS speeds is inversely proportional to the particle masses. For this example: it is given that $\left(\mathrm{n}_{1}, \mathrm{n}_{2}\right) N=(0.7,0.3) N,\left(M_{1}, M_{2}\right)=(0.9,0.1) M$, and the iteration results are $\left(v_{1, r m s}, v_{2, r m s}\right)=$ $(9.044,27.131),\left(v_{1, r m s}^{2}, v_{2, r m s}^{2}\right)=(81.79,736.09)$, and $\left(M_{1} v_{1, r m s}^{2}, M_{2} v_{2, r m s}^{2}\right)=(73.61,73.61) M$. These show that $M_{1} v_{1, r m s}^{2}=M_{2} v_{2, r m s}^{2}$. The results prove the Maxwell-Boltzmann speed distribution and also confirm Avogadro's law based on the mechanical behavior of the collisions of the particles. A proof of the Maxwell-Boltzmann speed distribution with analytical integration can be found in another paper by the authors (Lin et al., 2021). 

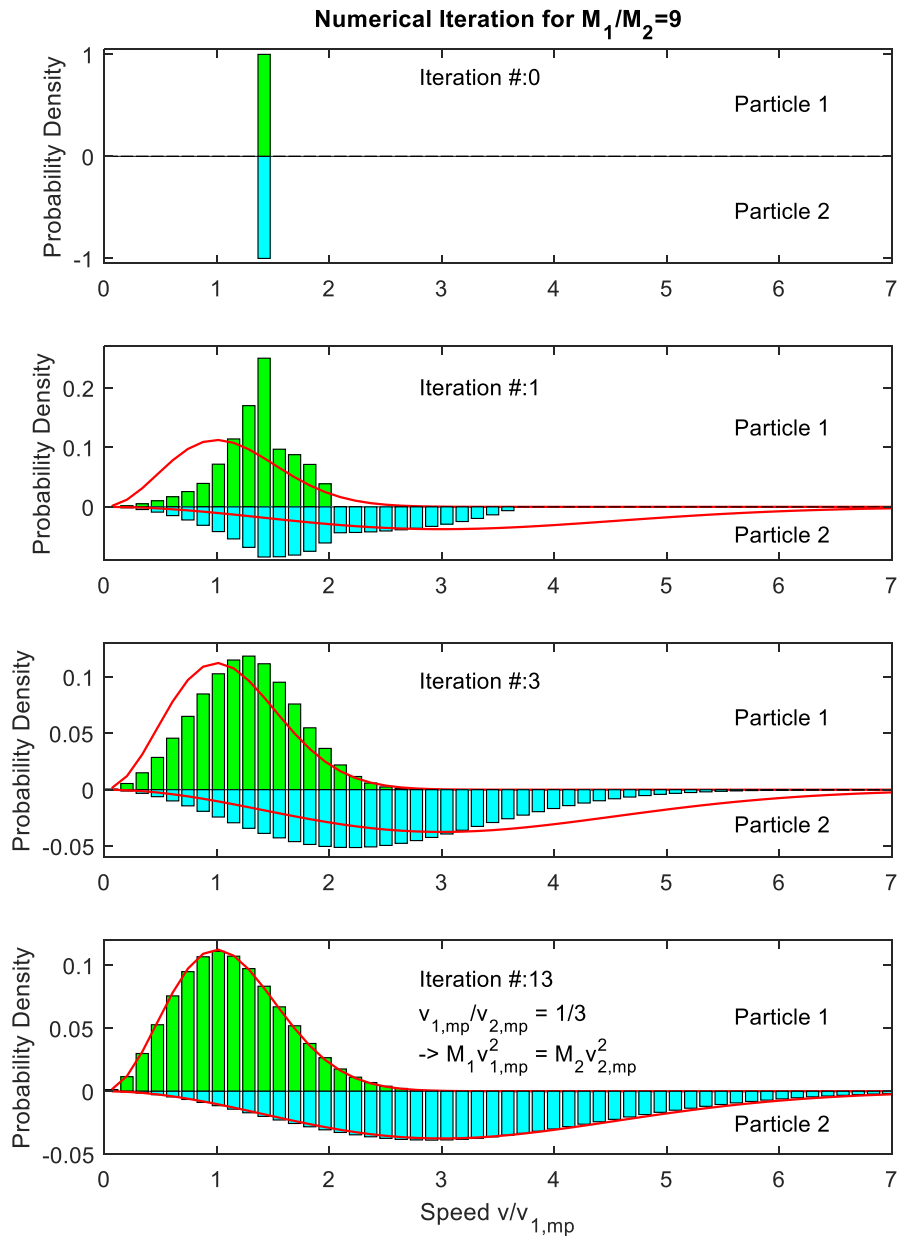

Figure 10. Thirteen iterations converge to the Maxwell-Boltzmann speed distribution

\section{Conclusion and Outlook}

It is essential to get the probability density function (PDF) $\psi^{a b}\left(u_{a} ; v_{a}, v_{b}\right)$ since from it, the Maxwell-Boltzmann speed distribution and the speed ratio can be obtained. From the derivation of the PDF $\psi^{a b}\left(u_{a} ; v_{a}, v_{b}\right)$, we reveal the basic mechanism behind the macroscopic phenomenon. The mechanics of the collision of particles is a bridge between microscopic behavior and macroscopic phenomenon. Another example is the macroscopic pressure and the microscopic velocity of the particles. We can see clearly that the universe does play dice with the random angles $\alpha$ and $\beta$ based on mechanics at the microscopic level for individual particles. By the way, on the other hand, group behaviors such as pressure waves or mass flows are deterministic at the macroscopic level.

The results, $M_{1} v_{1, r m s}^{2}=M_{2} v_{2, r m s}^{2}$, obtained from Figure 10, not only prove the Maxwell-Boltzmann speed distribution, but also Avogadro's law based on the mechanical behavior of the collisions of the particles.

This article gives mechanical proof of the Maxwell-Boltzmann speed distribution and the speed ratio for monatomic gases only. The same procedures can be applied to polyatomic gases. The PDF $\psi^{a b}\left(u_{a} ; v_{a}, v_{b}\right)$ must be extended to including the rotation (or spin) of molecules unless the rotation is small and its effect can be neglected. Moreover, the procedures may also be applied to charged particles. It is possible that the chemical characteristic of molecules could be revealed from the speed and spin distributions induced by the mutual action of two different kinds of molecules. Then, the unique procedure provided may have a significant impact on this kind of research.

\section{Acknowledgments}

We appreciate the words "Everything is mechanics" said by Chao-Chung Yu (1915 2014), late president of the National Taiwan University. Indeed, the mechanics are the foundation of the universe. We also appreciate Prof. Yu for his encouragement and support. 


\section{References}

Avogadro, A. (1811). Essai d'une maniere de determiner les masses relatives des molecules elementaires des corps, et les proportions selon lesquelles elles entrent dans ces combinations. Journal de Physique, 73, 58-76. English translation.

Boltzmann, L. (1872). Weitere studien uber das Warmegleichgewicht unter Gasmolekulen, Sitzungsbenchte der Kaiserlichen Akademie der Wissenschaften in Wien, Mathematisch-Naturwissenschaftliche Classe, 66, $275-370$.

Boltzmann, L. (1877). Über die Beziehung zwischen dem zweitenHauptsatz der mechanischen Wärmetheorie und der Wahrscheinlichkeitsrechnung respektive den Sätzen über das Wärmegleichgewicht." Sitzungsberichte der Kaiserlichen Akademie der Wissenschaften in Wien, Mathematisch-Naturwissenschaftliche Classe. Abt. II, 76, 1877, 373-435. Reprinted in Wissenschaftliche Abhandlungen, Vol. II, pp. 164-223, Leipzig: Barth, 1909. https://doi.org/10.1017/CBO9781139381437.011

Brush, S. G. (1966). Kinetic theory Vol.2. Irreversible processes. Pergamon Press, ISBN 9781483155937 1483155935.Selection.2:Ludwig Boltzmann:Further Studies on the Thermal Equilibrium of Gas Molecules (from Sitzungsbenchte der Kaiserlichen Akademie der Wissenschaften,Vienna, 1872), 88-175. https://doi.org/10.1016/B978-0-08-011869-7.50009-6

Garrod, C. (1995). Statistical mechanics and thermodynamics. University of California, Davis, New York Oxford: Oxford University Press, ISBN 0-19-508523-X (IBM software).

Herapath, J. (1821). Annals of Philosophy, Baldwin, Cradock, and Joy, 9, 273-293, 340-351, 401-416. Retrieved from https://books.google.com/books?id=nCsAAAAAMAAJ\&pg=RA1-PA273

Landau L. D., \& Lifshitz, E. M. (1969). Statistical physics. Institute of Physical Problems, U.S.S.R. Academy of Science, Vol.5 of Course of Theoretical Physic, Translated from the Russian by Peierls, E. and Peierls, R.F., London, Paris: Pergamon Press, ISBN 0-201-0-04167-7.

Lane, W. C. (2003). Temperature and pressure of an ideal gas: The equation of state. Physnet, Physics-Astronomy Dept., Mich. State Univ., 965-987. http://physnet.org/modules/pdfmodules/m156.pdf

Lin, H., \& Lin, T.-W. (2021). Mechanical Proof of the Maxwell-Boltzmann Speed Distribution With Analytical Integration, International Journal of Statistics and Probability, 10(3), 135-153. https://doi.org/10.5539/ijsp.v10n3p135

Lin, T.-W., \& Lin, H. (2019). Mechanical Proof of the Maxwell Speed Distribution, International Journal of Statistics and Probability, 8(2), 90-98. https://doi.org/10.5539/ijsp.v8n2p90

Maudlin, T. (2013). How to derive the equilibrium velocity distribution two ways (Neither of which is what you are expecting). Cosmology Summer School, Santa Cruz, July 26, 2013, NYU. Retrieved from http://hipacc.ucsc.edu/IPC2013/slides/130626_TimMaudlin.pdf

Maxwell, J. C. (1860a). V. Illustrations of the dynamical theory of gases, Part I. On the motions and collisions of perfectly elastic spheres, The London, Edinburgh, and Dubin Philosophical Magazine and Journal of Science, $4^{\text {th }}$ Series, 19, 19-32. https://doi.org/10.1080/14786446008642818

Maxwell, J. C. (1860b). II. Illustrations of the dynamical theory of gases, Part II. On the process of diffusion of two or more kinds of moving particles among one another, The London, Edinburgh, and Dubin Philosophical Magazine and Journal of Science, 4th Series, 20, 21-37. https://doi.org/10.1080/14786446008642902

Maxwell, J. C. (1867). XV. On the dynamical theory of gases, The London, Edinburgh, and Dubin Philosophical Magazine and Journal of Science, $4^{\text {th }}$ Series, 35, 129-145, \& $36, \quad 185-217$ https://doi.org/10.1080/14786446808639951

McQuarrie, D. A. (1976). Statistical mechanics. Indiana University, Harper Collins Publishers, ISBN 06-044366-9.

Waterston, J. J. (1843:1892). On the physics of media that are composed of free and perfectly elastic molecules in a state of motion. Philosophical Transactions of the Royal Society of London A, 183, 1-79 https://doi.org/10.1098/rsta.1892.0001

\section{Copyrights}

Copyright for this article is retained by the author(s), with first publication rights granted to the journal.

This is an open-access article distributed under the terms and conditions of the Creative Commons Attribution license (http://creativecommons.org/licenses/by/4.0/). 